\title{
THE TEICHMÜLLER SPACE OF GROUP INVARIANT SYMMETRIC STRUCTURES ON THE CIRCLE
}

\author{
Katsuhiko Matsuzaki \\ Waseda University, School of Education, Department of Mathematics \\ Shinjuku, Tokyo 169-8050, Japan; matsuzak@waseda.jp
}

\begin{abstract}
We introduce the quasisymmetric deformation space of a Fuchsian group $\Gamma$ within the group of symmetric self-homeomorphisms of the circle, and define this as the Teichmüller space $A T(\Gamma)$ of $\Gamma$-invariant symmetric structures. This is another generalization of the asymptotic Teichmüller space, and we verify the basic properties of this space. In particular, we show that $A T(\Gamma)$ is infinite dimensional, and in fact non-separable if $\Gamma$ admits a non-trivial deformation, even for a cofinite Fuchsian group $\Gamma$.
\end{abstract}

\section{Introduction}

All Teichmüller spaces of hyperbolic Riemann surfaces are embedded in the universal Teichmüller space $T$. This means that for any Fuchsian group $\Gamma$ that uniformizes a Riemann surface $R$, the Teichmüller space of $R$ can be represented as a closed subspace $T(\Gamma)$ of $T$, which consists of the fixed points of $\Gamma$. We consider a similar construction in the Teichmüller space $A T$ of symmetric structures on the circle $\mathbf{S}$. This space was first introduced by Gardiner and Sullivan [7]. In the language of $(G, X)$-structures, $T$ is regarded as the deformation space of $(\mathrm{Möb}(\mathbf{S}), \mathbf{S})$-structures, whereas $A T$ is the deformation space of (Sym, $\mathbf{S})$-structures. Here, $\operatorname{Möb}(\mathbf{S}) \cong \operatorname{PSL}(2, \mathbf{R})$ is the group of Möbius transformations of $\mathbf{S}$, and Sym is the larger group consisting of symmetric self-homeomorphisms of $\mathbf{S}$. Deformations are allowed within the category of the group QS of quasisymmetric self-homeomorphisms of $\mathbf{S}$.

The group Sym was introduced in [7], as the characteristic topological subgroup of QS consisting of all elements $g \in$ QS such that the adjoint map QS $\rightarrow$ QS defined by conjugation by $g$ is continuous at the identity. Subsequently, Earle, Gardiner, and Lakic [3] studied the asymptotic Teichmüller space $A T(R)$ for a Riemann surface $R$, which is a generalization of $A T$. This was introduced by focusing on the property that the quasiconformal extension to the unit disk $\mathbf{D}$ of a symmetric self-homeomorphism of $\mathbf{S}$ is asymptotically conformal; that is, its complex dilatation vanishes at the boundary $\mathbf{S}$.

In this paper, although we rely on the arguments providing the foundation of asymptotic Teichmüller spaces, we move in the different direction of investigating the Teichmüller space $A T(\Gamma)$ of $\Gamma$-invariant symmetric structures on $\mathbf{S}$, which is the closed subspace of $A T$ consisting of the fixed points of a Fuchsian group $\Gamma$. The major results demonstrated for $A T(\Gamma)$ in this paper are summarized as follows.

https://doi.org/10.5186/aasfm.2017.4235

2010 Mathematics Subject Classification: Primary 30F60; Secondary 32G15.

Key words: Quasiconformal, quasisymmetric, asymptotic Teichmüller space, asymptotic Bers embedding, barycentric extension, complex Banach manifold.

This work was supported by JSPS KAKENHI 25287021 . 
Theorem 1.1. For a Fuchsian group $\Gamma$, the Teichmüller space $A T(\Gamma)$ of $\Gamma$ invariant symmetric structures on $\mathbf{S}$, endowed with the asymptotic Teichmüller distance, has a complex non-separable Banach manifold structure if $T(\Gamma)$ is non-trivial. Moreover, $A T(\Gamma)$ is contractible.

Here, the properties other than the infinite dimensionality of $A T(\Gamma)$ are standard for Teichmüller spaces, and are obtained by modifying existing arguments in the literature. In particular, we utilize certain results that Earle, Markovic, and Saric [5] demonstrated using the barycentric extension of quasisymmetric selfhomeomorphisms. On the other hand, the infinite dimensionality of $A T(\Gamma)$ requires the application of a novel technique. In fact, $A T(\Gamma)$ is not separable even if $\Gamma$ is cofinite. The proof proceeds by refining the arguments given in our previous papers $[10,11]$.

The importance of $A T(\Gamma)$ arises when we consider the quasisymmetric deformation space of a Fuchsian group $\Gamma$ in the group of certain automorphisms of $\mathbf{S}$. By definition, $A T(\Gamma)$ itself is the deformation space of $\Gamma$ in Sym. The key feature of this space is that other deformation spaces of $\Gamma$ can be embedded in $A T(\Gamma)$. Besides the fact that the Teichmüller space $T(\Gamma)$ is embedded in $A T(\Gamma)$ (this will be seen in Proposition 5.1), we have shown in [14] that the deformation space of $\Gamma$ in the group of diffeomorphisms of $\mathbf{S}$ with Hölder continuous derivatives is embedded in $A T(\Gamma)$. Then, we can understand the rigidity of such a deformation through the coincidence of these deformation spaces in the ambient space $A T(\Gamma)$.

Fundamental results concerning quasiconformal maps and Teichmüller spaces can be found in the standard monographs $[1,6,8,16]$.

\section{A new asymptotic Teichmüller space}

We denote the group of all quasiconformal self-homeomorphisms of $\mathbf{D}$ by $\mathrm{QC}(\mathbf{D})$. For $w \in \mathrm{QC}(\mathbf{D})$, we set its complex dilatation as $\mu_{w}(z)=w_{\bar{z}} / w_{z}$. Every quasiconformal self-homeomorphism $w$ of $\mathbf{D}$ extends to a self-homeomorphism $f$ of $\mathbf{S}$. We define such an $f$ to be quasisymmetric, and denote the group of all quasisymmetric self-homeomorphisms of $\mathbf{S}$ by QS. This correspondence gives rise to a surjective homomorphism $q$ : QC(D) $\rightarrow$ QS. The topology on QS is defined by using the quasisymmetric constant. See [7, Section 1].

The universal Teichmüller space is defined by $T=\operatorname{Möb}(\mathbf{S}) \backslash \mathrm{QS}$, with its elements represented by the equivalence classes $[f]$ for $f \in$ QS. Through the solution of the Beltrami equation (the measurable Riemann mapping theorem, see [1]), the space of Beltrami coefficients

$$
\operatorname{Bel}(\mathbf{D})=\left\{\mu \in L^{\infty}(\mathbf{D}) \mid\|\mu\|_{\infty}<1\right\}
$$

is identified with $\mathrm{QC}(\mathbf{D})$ modulo post-composition of $\operatorname{Möb}(\mathbf{D})$. Then, the boundary extension map $q$ gives rise to a well-defined surjection

$$
\pi: \operatorname{Bel}(\mathbf{D}) \cong \operatorname{Möb}(\mathbf{D}) \backslash \mathrm{QC}(\mathbf{D}) \longrightarrow T=\operatorname{Möb}(\mathbf{S}) \backslash \mathrm{QS},
$$

which is called the Teichmüller projection.

The topology on $T$ is the quotient topology inherited from $\operatorname{Bel}(\mathbf{D})$ through $\pi$. We note that this coincides with the quotient topology arising from QS. Alternatively, this can be introduced as follows. The open unit ball $\operatorname{Bel}(\mathbf{D})$ of $L^{\infty}(\mathbf{D})$ is endowed with the hyperbolic distance:

$$
d_{h}(\mu, \nu)=\log \left(1+\left\|\frac{\mu-\nu}{1-\bar{\nu} \mu}\right\|_{\infty}\right)-\log \left(1-\left\|\frac{\mu-\nu}{1-\bar{\nu} \mu}\right\|_{\infty}\right) \quad(\mu, \nu \in \operatorname{Bel}(\mathbf{D})) .
$$


Then, the quotient pseudo-distance on $T$ induced by $\pi$ is in fact a distance, which is called the Teichmüller distance $d_{T}$. The topology on $T$ induced by $d_{T}$ coincides with the quotient topology arising from $\operatorname{Bel}(\mathbf{D})$.

The group QS acts on $T$ canonically, as follows:

$$
([f], g) \in T \times \mathrm{QS} \mapsto g^{*}[f]:=[f \circ g] \in T .
$$

Clearly, the action is faithful and transitive. Moreover, this is isometric with respect to $d_{T}$. The isotropy subgroup of QS at the origin [id] $\in T$ coincides with $\operatorname{Möb}(\mathbf{S})$. The condition that $g \in \mathrm{QS}$ fixes $[f] \in T$, that is, $g^{*}[f]=[f]$, can be written as $\left[f g f^{-1}\right]=[\mathrm{id}]$, and this is equivalent to the condition that $f g f^{-1} \in \operatorname{Möb}(\mathbf{S})$.

A quasiconformal self-homeomorphism $w \in \mathrm{QC}(\mathbf{D})$ is called asymptotically conformal if the complex dilatation $\mu_{w}$ vanishes at the boundary $\mathbf{S}$; that is, $\mu_{w}(z) \rightarrow$ $0(|z| \rightarrow 1)$. The closed subspace of $\operatorname{Bel}(\mathbf{D})$ consisting of all Beltrami coefficients vanishing at the boundary is denoted by $\operatorname{Bel}_{0}(\mathbf{D})$, and the subgroup of $\mathrm{QC}(\mathbf{D})$ consisting of all asymptotically conformal automorphisms of $\mathbf{D}$ is denoted by AC(D). On the other hand, a quasisymmetric self-homeomorphism $f \in$ QS can be defined to be symmetric if there is some $w \in \mathrm{AC}(\mathbf{D})$ such that $q(w)=f$. The group of all symmetric self-homeomorphisms of $\mathbf{S}$ is denoted by Sym. Then, the restriction of the boundary extension map $q$ to $\mathrm{AC}(\mathbf{D})$ gives rise to a surjective homomorphism $q: \mathrm{AC}(\mathbf{D}) \rightarrow$ Sym.

Gardiner and Sullivan [7] studied the asymptotic Teichmüller space defined by

$$
A T=\mathrm{Sym} \backslash \mathrm{QS} .
$$

The elements of this space are represented by the equivalence classes $[[f]]$ for $f \in$ QS. In addition, the little universal Teichmüller space is defined by

$$
T_{0}=\operatorname{Möb}(\mathbf{S}) \backslash \operatorname{Sym}=\pi\left(\operatorname{Bel}_{0}(\mathbf{D})\right) \subset T .
$$

There exists a canonical projection

$$
\alpha: T \rightarrow A T
$$

given by $[f] \mapsto[[f]]$. The quotient topology of $A T$ is induced from $T$ by $\alpha$. Furthermore, because $T_{0} \subset T$ (Sym $\subset$ QS) is closed which was shown in [7, Corollary 2.1], and as QS acts isometrically with respect to $d_{T}$ and transitively on $T$, we see that the quotient pseudo-distance on $A T$ induced by $\alpha$ from $d_{T}$ is in fact a distance $d_{A T}$. We call this the asymptotic Teichmüller distance.

The group QS acts on AT by

$$
([[f]], g) \in A T \times \mathrm{QS} \mapsto g^{*}[[f]]:=[[f \circ g]] \in A T .
$$

This is well-defined, because the action of QS on $T$ sends each fiber of the projection $\alpha$ onto another fiber. This action is isometric with respect to $d_{A T}$. The isotropy subgroup of QS at the origin $[[i d]] \in A T$ coincides with Sym.

The deformation space of a Fuchsian group $\Gamma$ in $\operatorname{Möb}(\mathbf{S})$ is given as the Teichmüller space of $\Gamma$, which is defined by

$$
T(\Gamma)=\operatorname{Möb}(\mathbf{S}) \backslash\left\{f \in \mathrm{QS} \mid f \Gamma f^{-1} \subset \operatorname{Möb}(\mathbf{S})\right\} \subset T .
$$

This can be alternatively written as

$$
T(\Gamma)=\left\{[f] \in T \mid \gamma^{*}[f]=[f](\forall \gamma \in \Gamma)\right\},
$$

and hence we see that $T(\Gamma)$ is a closed subspace of $T$. The topology on $T(\Gamma)$ is given by the relative topology as a subspace of $T$. We can also consider the inner distance 
on $T(\Gamma)$ induced from $d_{T}$ compatible with this topology, although in general this distance differs from the ordinary Teichmüller distance on $T(R)$ for $R=\mathbf{D} / \Gamma$.

In a similar manner, we can introduce the following space, which is our main object of study in this paper.

Definition. The deformation space of a Fuchsian group $\Gamma \subset \operatorname{Möb}(\mathbf{S})$ in Sym is defined by

$$
A T(\Gamma)=\operatorname{Sym} \backslash\left\{f \in \mathrm{QS} \mid f \Gamma f^{-1} \subset \operatorname{Sym}\right\}=\left\{[[f]] \in A T \mid \gamma^{*}[[f]]=[[f]](\forall \gamma \in \Gamma)\right\},
$$

which is a closed subspace of $A T$. The topology on $A T(\Gamma)$ is the relative topology as a subspace of $A T$, and the inner distance $d_{A T(\Gamma)}$ induced from $d_{A T}$ can be given as the asymptotic Teichmüller distance on $A T(\Gamma)$.

We remark that $A T(\Gamma)$ is different from the asymptotic Teichmüller space $A T(R)$ of the Riemann surface $R=\mathbf{D} / \Gamma$. We call $A T(\Gamma)$ the asymptotic Teichmüller space for a Fuchsian group $\Gamma$, or in order to distinguish it from that of $R$, the Teichmüller space of $\Gamma$-invariant symmetric structures on $\mathbf{S}$.

Obviously, the inclusion relation of Fuchsian groups $\Gamma_{1} \subset \Gamma_{2}$ implies that $A T\left(\Gamma_{1}\right)$ $\supset A T\left(\Gamma_{2}\right)$ and $d_{A T\left(\Gamma_{1}\right)} \leq d_{A T\left(\Gamma_{2}\right)}$. The same property is satisfied for the Teichmüller space $T(\Gamma)$ for a Fuchsian group $\Gamma$. Here, we can raise the following questions for future study:

(1) Does $A T\left(\Gamma_{1}\right)=A T\left(\Gamma_{2}\right)$ imply that $\Gamma_{1}=\Gamma_{2}$ ?

(2) Does $d_{A T\left(\Gamma_{1}\right)}=d_{A T\left(\Gamma_{2}\right)}$ on $A T\left(\Gamma_{2}\right)$ imply that $\Gamma_{1}=\Gamma_{2}$, or that $\Gamma_{2} / \Gamma_{1}$ is small in some sense? More generally, compare $d_{A T\left(\Gamma_{2}\right)}$ with $d_{A T\left(\Gamma_{1}\right)}$ restricted to $A T\left(\Gamma_{2}\right)$.

Concerning these problems for the Teichmüller spaces $T(\Gamma)$ and $T(R)$, we refer the reader to [9], [8, Section V.3.2], and McMullen [15].

\section{Complex Banach manifold structure}

In this section, we introduce a complex Banach manifold structure to the Teichmüller space $A T(\Gamma)$ of $\Gamma$-invariant symmetric structures on $\mathbf{S}$ for a Fuchsian group $\Gamma$. This will be achieved by using the asymptotic Bers embedding. We first review the construction of this mapping, and then modify the argument for our present case.

We consider the complex Banach space of hyperbolically bounded holomorphic functions (quadratic differentials) on $\mathbf{D}^{*}=\widehat{\mathbf{C}}-\overline{\mathbf{D}}$ :

$$
B\left(\mathbf{D}^{*}\right)=\left\{\varphi \in \operatorname{Hol}\left(\mathbf{D}^{*}\right)\left|\|\varphi\|_{\infty}=\sup _{z \in \mathbf{D}^{*}} \rho_{\mathbf{D}^{*}}^{-2}(z)\right| \varphi(z) \mid<\infty\right\} .
$$

Here, $\rho_{\mathbf{D}^{*}}$ is the hyperbolic density on $\mathbf{D}^{*}$. The Bers projection $\Phi: \operatorname{Bel}(\mathbf{D}) \rightarrow B\left(\mathbf{D}^{*}\right)$ with correspondence $\mu \mapsto \varphi$ is defined by taking the Schwarzian derivative $\varphi$ of a conformal homeomorphism of $\mathbf{D}^{*}$ that extends to a quasiconformal homeomorphism of $\mathbf{D}$, having the complex dilatation $\mu$. Then, we have a well-defined injection $\beta: T \rightarrow$ $B\left(\mathbf{D}^{*}\right)$, given by $\Phi \circ \pi^{-1}$, which is called the Bers embedding. This can be shown to be a homeomorphism onto a bounded domain in $B\left(\mathbf{D}^{*}\right)$, and hence provides a complex Banach manifold structure for $T$.

The group Möb $\left(\mathbf{D}^{*}\right)$ of Möbius transformations of $\mathbf{D}^{*}$ acts on the complex Banach space $B\left(\mathbf{D}^{*}\right)$ linear isometrically, as

$$
(\varphi, g) \in B\left(\mathbf{D}^{*}\right) \times \operatorname{Möb}\left(\mathbf{D}^{*}\right) \mapsto g^{*} \varphi \in B\left(\mathbf{D}^{*}\right) ; \quad\left(g^{*} \varphi\right)(z):=\varphi(g(z)) g^{\prime}(z)^{2} .
$$

It is easy to see that under the Bers embedding $\beta$ and the identification $\operatorname{Möb}(\mathbf{S}) \cong$ $\operatorname{Möb}\left(\mathbf{D}^{*}\right)$, the action of $\operatorname{Möb}(\mathbf{S})$ on $T$ and the action of $\operatorname{Möb}\left(\mathbf{D}^{*}\right)$ on $B\left(\mathbf{D}^{*}\right)$ are 
equivalent. More precisely, we have that $\beta \circ g^{*}=g^{*} \circ \beta$ on $T$, for every $g \in \operatorname{Möb}(\mathbf{S}) \cong$ $\operatorname{Möb}\left(\mathbf{D}^{*}\right)$.

For a Fuchsian group $\Gamma$, regarded as a subgroup of $\mathrm{Möb}\left(\mathbf{D}^{*}\right)$, let $B\left(\mathbf{D}^{*}, \Gamma\right)$ be the closed subspace of $B\left(\mathbf{D}^{*}\right)$ consisting of all elements fixed by every $\gamma \in \Gamma$. Then, the Bers embedding $\beta: T \rightarrow B\left(\mathbf{D}^{*}\right)$ restricted to the closed subspace $T(\Gamma)$ is a homeomorphism onto the bounded domain $\beta(T) \cap B\left(\mathbf{D}^{*}, \Gamma\right)$ in the complex Banach space $B\left(\mathbf{D}^{*}, \Gamma\right)$. Verifying the inclusion $\beta(T(\Gamma)) \subset \beta(T) \cap B\left(\mathbf{D}^{*}, \Gamma\right)$ is easier, but demonstrating the coincidence is more difficult. For example, see [5, Corollary 8.1] and [8, Section V.4.4]. In particular, $T(\Gamma)$ admits a complex Banach manifold structure modeled on $B\left(\mathbf{D}^{*}, \Gamma\right)$.

Following the case of ordinary Teichmüller spaces, we introduce a complex structure to $A T(\Gamma)$. Let $B_{0}\left(\mathbf{D}^{*}\right)$ be the closed subspace consisting of all elements $\varphi \in$ $B\left(\mathbf{D}^{*}\right)$ that vanish at the boundary $\mathbf{S}$, which means that $\lim _{|z| \rightarrow 1} \rho_{\mathbf{D}^{*}}^{-2}(z)|\varphi(z)|=0$. Then, it was verified by Gardiner and Sullivan [7, Theorem 4.1] and Earle, Gardiner, and Lakic [3, Corollary 2.10] that the Bers embedding $\beta$ satisfies $\beta\left(T_{0}\right) \subset B_{0}\left(\mathbf{D}^{*}\right)$, and moreover $\beta$ projects down to a well-defined map

$$
\widehat{\beta}: A T=\operatorname{Sym} \backslash \mathrm{QS} \rightarrow B_{0}\left(\mathbf{D}^{*}\right) \backslash B\left(\mathbf{D}^{*}\right)
$$

into the quotient Banach space $\widehat{B}\left(\mathbf{D}^{*}\right)=B_{0}\left(\mathbf{D}^{*}\right) \backslash B\left(\mathbf{D}^{*}\right)$. We call this map the asymptotic Bers embedding.

The equivalence class of $\varphi \in B\left(\mathbf{D}^{*}\right)$ is denoted by $[\varphi]_{B} \in \widehat{B}\left(\mathbf{D}^{*}\right)$, and the projection $P_{B}: B\left(\mathbf{D}^{*}\right) \rightarrow \widehat{B}\left(\mathbf{D}^{*}\right)$ is given by $P_{B}(\varphi)=[\varphi]_{B}$. Then, this satisfies the condition that

$$
P_{B} \circ \beta=\widehat{\beta} \circ \alpha
$$

on $T$, for the projection $\alpha: T \rightarrow A T$. Furthermore, $\widehat{\beta}$ is a homeomorphism onto a bounded domain in $\widehat{B}\left(\mathbf{D}^{*}\right)$, and in particular, $\widehat{\beta}$ is injective (see [5, Theorem 1] and [6, Section 16.8]). This fact can be explained by Lemma 4.2 given in Section 4 below. From this, we can see that $A T$ admits a complex Banach manifold structure modeled on $\widehat{B}\left(\mathbf{D}^{*}\right)$.

The group $\operatorname{Möb}\left(\mathbf{D}^{*}\right)$ acts on $\widehat{B}\left(\mathbf{D}^{*}\right)$ linear isometrically. This is because $\operatorname{Möb}\left(\mathbf{D}^{*}\right)$ acts on $B\left(\mathbf{D}^{*}\right)$, keeping $B_{0}\left(\mathbf{D}^{*}\right)$ invariant. Then, by the fact that $\beta \circ g^{*}=g^{*} \circ \beta$ and $P_{B} \circ \beta=\widehat{\beta} \circ \alpha$ on $T$, we see that

$$
\widehat{\beta} \circ g^{*}=g^{*} \circ \widehat{\beta}
$$

on $A T$, for every $g \in \operatorname{Möb}(\mathbf{S}) \cong \operatorname{Möb}\left(\mathbf{D}^{*}\right)$.

For a Fuchsian group $\Gamma \subset \operatorname{Möb}\left(\mathbf{D}^{*}\right)$, we consider its linear isometric action on $\widehat{B}\left(\mathbf{D}^{*}\right)$. Let $\widehat{B}\left(\mathbf{D}^{*}, \Gamma\right)$ be the closed subspace of $\widehat{B}\left(\mathbf{D}^{*}\right)$ consisting of all elements fixed by every $\gamma \in \Gamma$. In other words,

$$
\widehat{B}\left(\mathbf{D}^{*}, \Gamma\right)=\left\{[\varphi]_{B} \in \widehat{B}\left(\mathbf{D}^{*}\right) \mid\left[\gamma^{*} \varphi\right]_{B}=[\varphi]_{B}(\forall \gamma \in \Gamma)\right\} .
$$

Note that $\widehat{B}\left(\mathbf{D}^{*}, \Gamma\right)$ is different from the projection of $B\left(\mathbf{D}^{*}, \Gamma\right)$ by $P_{B}$. In fact, the former contains the latter as a proper subspace in many cases. This will be seen later in Section 5.

The asymptotic Bers embedding $\widehat{\beta}: A T \rightarrow \widehat{B}\left(\mathbf{D}^{*}\right)$ restricted to the closed subspace $A T(\Gamma)$ is a homeomorphism onto the image in $\widehat{\beta}(A T) \cap \widehat{B}\left(\mathbf{D}^{*}, \Gamma\right)$. In fact, we will prove later that

$$
\widehat{\beta}(A T(\Gamma))=\widehat{\beta}(A T) \cap \widehat{B}\left(\mathbf{D}^{*}, \Gamma\right) .
$$


In this manner, we furnish $A T(\Gamma)$ with a complex Banach manifold structure modeled on $\widehat{B}\left(\mathbf{D}^{*}, \Gamma\right)$.

Now, we summarize the contents of this section as a theorem.

Theorem 3.1. The restriction of the asymptotic Bers embedding $\widehat{\beta}: A T \rightarrow$ $\widehat{B}\left(\mathbf{D}^{*}\right)$ to the Teichmüller space $A T(\Gamma)$ of $\Gamma$-invariant symmetric structures on $\mathbf{S}$ is a homeomorphism onto the bounded domain $\widehat{\beta}(A T) \cap \widehat{B}\left(\mathbf{D}^{*}, \Gamma\right)$. Hence, $A T(\Gamma)$ admits a complex Banach manifold structure modeled on $\widehat{B}\left(\mathbf{D}^{*}, \Gamma\right)$.

Proof. The only statement for which a proof is required is that $\widehat{\beta}(A T(\Gamma))=$ $\widehat{\beta}(A T) \cap \widehat{B}\left(\mathbf{D}^{*}, \Gamma\right)$. This will be provided in Lemma 4.4 .

Remark. As in the case of $A T$, we can also consider the problem of whether or not the Kobayashi distance given by the complex structure on $A T(\Gamma)$ coincides with the asymptotic Teichmüller distance $d_{A T(\Gamma)}$. See [4, Section 3.2].

\section{Barycentric section and contractibility}

In this section, we show that the Teichmüller space $A T(\Gamma)$ of $\Gamma$-invariant symmetric structures on $\mathbf{S}$ is contractible as a topological space. This is a common property of Teichmüller spaces, and is usually verified by demonstrating the existence of a global continuous section for the Teichmüller or the Bers projections. The barycentric section induced by the barycentric extension of quasisymmetric self-homeomorphisms of $\mathbf{S}$ is appropriate for this purpose. Then, the contractibility of the open unit ball of relevant Beltrami coefficients provides the solution.

Douady and Earle [2] introduced the barycentric extension e: $\mathrm{QS} \rightarrow \mathrm{QC}(\mathbf{D})$, as a section for the boundary extension map $q$ : QC(D) $\rightarrow$ QS satisfying $q \circ e=$ id. Moreover, $e$ is conformally natural, in the sense that

$$
\text { (1) } e\left(g_{1} \circ f\right)=e\left(g_{1}\right) \circ e(f) ; \quad(2) \quad e\left(f \circ g_{2}\right)=e(f) \circ e\left(g_{2}\right)
$$

for any $g_{1}, g_{2} \in \operatorname{Möb}(\mathbf{S})$ and any $f \in$ QS. Then, by (1) we can divide both sides of $e$ by $\operatorname{Möb}(\mathbf{S}) \cong \operatorname{Möb}(\mathbf{D})$, obtaining a section $s^{\prime}: T \rightarrow \operatorname{Bel}(\mathbf{D})$ for the Teichmüller projection $\pi: \operatorname{Bel}(\mathbf{D}) \rightarrow T$. By the composition of $s^{\prime}$ with the inverse of the Bers embedding $\beta$, we obtain $s: \beta(T) \rightarrow \operatorname{Bel}(\mathbf{D})$, satisfying $\Phi \circ s=$ id for the Bers projection $\Phi: \operatorname{Bel}(\mathbf{D}) \rightarrow \beta(T)$. We call this $s$ the barycentric section, and it provides a real analytic section for $\Phi$.

Let $L^{\infty}(\mathbf{D})$ be the complex Banach space of bounded measurable functions $\mu$ on $\mathbf{D}$, with supremum norm $\|\mu\|_{\infty}$. We call an element of $L^{\infty}(\mathbf{D})$ a Beltrami differential. Then, $\operatorname{Bel}(\mathbf{D})$ is the open unit ball on this space. Let $L_{0}^{\infty}(\mathbf{D})$ be the closed subspace of $L^{\infty}(\mathbf{D})$ consisting of all functions $\mu$ vanishing at the boundary $\mathbf{S}$. Then, $\operatorname{Bel}_{0}(\mathbf{D})=\operatorname{Bel}(\mathbf{D}) \cap L_{0}^{\infty}(\mathbf{D})$. Moreover, we consider the quotient Banach space $\widehat{L}^{\infty}(\mathbf{D})=L_{0}^{\infty}(\mathbf{D}) \backslash L^{\infty}(\mathbf{D})$ and its open unit ball $\widehat{\operatorname{Bel}}(\mathbf{D})$, which is given by $\operatorname{Bel}(\mathbf{D})$ modulo $L_{0}^{\infty}(\mathbf{D})$. We denote the equivalence class of $\mu \in L^{\infty}(\mathbf{D})$ by $[\mu]_{L} \in \widehat{L}^{\infty}(\mathbf{D})$, and the projection $P_{L}: L^{\infty}(\mathbf{D}) \rightarrow \widehat{L}^{\infty}(\mathbf{D})$ is given by $P_{L}(\mu)=[\mu]_{L}$.

A basic property concerning the projections $P_{L}: \operatorname{Bel}(\mathbf{D}) \rightarrow \widehat{\operatorname{Bel}}(\mathbf{D})$ and $\alpha: T \rightarrow$ $A T$ is given as follows, which was shown in [3, Lemma 2.9] and [5, Corollary 2.1]. From this, we obtain the asymptotic Teichmüller projection $\widehat{\pi}: \widehat{\operatorname{Bel}}(\mathbf{D}) \rightarrow A T$ which satisfies

$$
\widehat{\pi} \circ P_{L}=\alpha \circ \pi
$$

on $\operatorname{Bel}(\mathbf{D})$. 
Proposition 4.1. For $\mu_{1}, \mu_{2} \in \operatorname{Bel}(\mathbf{D})$, if $P_{L}\left(\mu_{1}\right)=P_{L}\left(\mu_{2}\right)$, then $\alpha \circ \pi\left(\mu_{1}\right)=$ $\alpha \circ \pi\left(\mu_{2}\right)$ in $A T$.

The Bers projection $\Phi: \operatorname{Bel}(\mathbf{D}) \rightarrow B\left(\mathbf{D}^{*}\right)$ is a holomorphic split submersion onto $\beta(T)$. For details concerning holomorphic split submersions, see [16, Section 1.6.5]. This satisfies $\Phi\left(\operatorname{Bel}_{0}(\mathbf{D})\right) \subset B_{0}\left(\mathbf{D}^{*}\right)$, as in [7], and moreover it has been proved in [3, Theorem 2.3] that $\Phi$ projects down to a holomorphic split submersion $\widehat{\Phi}: \widehat{\operatorname{Bel}}(\mathbf{D}) \rightarrow$ $\widehat{B}\left(\mathbf{D}^{*}\right)$ onto $\widehat{\beta}(A T)$, which we call the asymptotic Bers projection. This satisfies

$$
\widehat{\Phi} \circ P_{L}=P_{B} \circ \Phi
$$

on $\operatorname{Bel}(\mathbf{D})$, and hence $\widehat{\beta} \circ \widehat{\pi}=\widehat{\Phi}$ on $\widehat{\operatorname{Bel}}(\mathbf{D})$.

The next lemma was proved by Earle, Markovic, and Saric [5, Theorem 4]. As an additional formulation of the essential part of this claim, we see that the barycentric extension $e$ satisfies asymptotically conformal naturality:

$$
e(\gamma \circ g) \circ e(g)^{-1} \in \mathrm{AC}(\mathbf{D})
$$

for every $g \in$ QS and every $\gamma \in$ Sym.

Lemma 4.2. For $\mu_{1}, \mu_{2} \in \operatorname{Bel}(\mathbf{D})$, the following conditions are equivalent:

(1) $\alpha \circ \pi\left(\mu_{1}\right)=\alpha \circ \pi\left(\mu_{2}\right)$ in $A T$;

(2) $P_{B} \circ \Phi\left(\mu_{1}\right)=P_{B} \circ \Phi\left(\mu_{2}\right)$ in $\widehat{B}\left(\mathbf{D}^{*}\right)$;

(3) $P_{L} \circ s \circ \Phi\left(\mu_{1}\right)=P_{L} \circ s \circ \Phi\left(\mu_{2}\right)$ in $\widehat{\operatorname{Bel}}(\mathbf{D})$.

In virtue of this property, the barycentric section $s$ descends to the asymptotic barycentric section $\widehat{s}: \widehat{\beta}(A T) \rightarrow \widehat{\operatorname{Bel}}(\mathbf{D})$, which is a real analytic section for $\widehat{\Phi}$, as in [5, Theorem 5].

The group Möb(D) of Möbius transformations of $\mathbf{D}$ acts on the complex Banach space $L^{\infty}(\mathbf{D})$ linear isometrically, as

$$
(\mu, g) \in L^{\infty}(\mathbf{D}) \times \operatorname{Möb}(\mathbf{D}) \mapsto g^{*} \mu \in L^{\infty}(\mathbf{D}) ; \quad\left(g^{*} \mu\right)(z):=\mu(g(z)) \frac{\overline{g^{\prime}(z)}}{g^{\prime}(z)} .
$$

This action keeps $L_{0}^{\infty}(\mathbf{D})$ invariant. Hence, it induces a linear isometric action of $\operatorname{Möb}(\mathbf{D})$ on $\widehat{L}^{\infty}(\mathbf{D})$, and we have that $g^{*}: \widehat{L}^{\infty}(\mathbf{D}) \rightarrow \widehat{L}^{\infty}(\mathbf{D})$ for each $g \in \operatorname{Möb}(\mathbf{D})$. Let $L^{\infty}(\mathbf{D}, \Gamma)$ be the closed subspace of $L^{\infty}(\mathbf{D})$ consisting of all elements that are fixed by every element $\gamma$ of a Fuchsian group $\Gamma$, and let $\widehat{L}^{\infty}(\mathbf{D}, \Gamma)$ be the closed subspace of $\widehat{L}^{\infty}(\mathbf{D})$ consisting of all elements fixed by every $\gamma \in \Gamma$. The open unit balls on these spaces are denoted by $\operatorname{Bel}(\mathbf{D}, \Gamma)$ and $\widehat{\operatorname{Bel}}(\mathbf{D}, \Gamma)$, respectively.

By the fact that $\pi \circ g^{*}=g^{*} \circ \pi$ on $\operatorname{Bel}(\mathbf{D})$ for every $g \in \operatorname{Möb}(\mathbf{D}) \cong \operatorname{Möb}(\mathbf{S})$, the Teichmüller projection gives $\pi: \operatorname{Bel}(\mathbf{D}, \Gamma) \rightarrow T(\Gamma)$ for a Fuchsian group $\Gamma$. In addition, because $\Phi \circ g^{*}=g^{*} \circ \Phi$ on $\operatorname{Bel}(\mathbf{D})$ for every $g \in \operatorname{Möb}(\mathbf{D}) \cong \operatorname{Möb}\left(\mathbf{D}^{*}\right)$, the Bers projection gives $\Phi: \operatorname{Bel}(\mathbf{D}, \Gamma) \rightarrow B\left(\mathbf{D}^{*}, \Gamma\right)$. Similarly, we can prove the following.

Proposition 4.3. The asymptotic Teichmüller projection $\widehat{\pi}: \widehat{\operatorname{Bel}}(\mathbf{D}) \rightarrow A T$ satisfies $\widehat{\pi}(\widehat{\operatorname{Bel}}(\mathbf{D}, \Gamma)) \subset A T(\Gamma)$, and the asymptotic Bers projection $\widehat{\Phi}: \widehat{\operatorname{Bel}}(\mathbf{D}) \rightarrow$ $\widehat{B}\left(\mathbf{D}^{*}\right)$ satisfies $\widehat{\Phi}(\widehat{\operatorname{Bel}}(\mathbf{D}, \Gamma)) \subset \widehat{B}\left(\mathbf{D}^{*}, \Gamma\right)$, for any Fuchsian group $\Gamma$.

Proof. Because $\widehat{\pi} \circ P_{L}=\alpha \circ \pi$ on $\operatorname{Bel}(\mathbf{D})$, we see from $\pi \circ g^{*}=g^{*} \circ \pi$ that

$$
\widehat{\pi} \circ g^{*}=g^{*} \circ \widehat{\pi}
$$


on $\widehat{\operatorname{Bel}}(\mathbf{D})$, for every $g \in \operatorname{Möb}(\mathbf{D}) \cong \operatorname{Möb}(\mathbf{S})$. This yields the inclusion $\widehat{\pi}(\widehat{\operatorname{Bel}}(\mathbf{D}, \Gamma)) \subset$ $A T(\Gamma)$. Similarly, because $\widehat{\Phi} \circ P_{L}=P_{B} \circ \Phi$ on $\operatorname{Bel}(\mathbf{D})$, we see from $\Phi \circ g^{*}=g^{*} \circ \Phi$ that

$$
\widehat{\Phi} \circ g^{*}=g^{*} \circ \widehat{\Phi}
$$

for every $g \in \operatorname{Möb}(\mathbf{D}) \cong \operatorname{Möb}\left(\mathbf{D}^{*}\right)$. This yields the inclusion $\widehat{\Phi}(\widehat{\operatorname{Bel}}(\mathbf{D}, \Gamma)) \subset$ $\widehat{B}\left(\mathbf{D}^{*}, \Gamma\right)$.

From the first statement of the above proposition and $\widehat{\beta} \circ \widehat{\pi}=\widehat{\Phi}$, we see that the image $\widehat{\Phi}(\widehat{\operatorname{Bel}}(\mathbf{D}, \Gamma))$ is contained in $\widehat{\beta}(A T(\Gamma))$. On the other hand, by considering $\widehat{\beta} \circ g^{*}=g^{*} \circ \widehat{\beta}$ on $A T$, we can show that $\widehat{\beta}(A T(\Gamma))$ is contained in $\widehat{\beta}(A T) \cap \widehat{B}\left(\mathbf{D}^{*}, \Gamma\right)$.

Several properties of the barycentric section arise from the conformal naturality of the barycentric extension $e$. We see from conformal naturality $(2)$ that the restriction of the barycentric section $s$ to $\beta(T) \cap B\left(\mathbf{D}^{*}, \Gamma\right)$ provides a real analytic section for $\Phi: \operatorname{Bel}(\mathbf{D}, \Gamma) \rightarrow \beta(T(\Gamma))$. Similarly, by considering both the conformal naturality and the asymptotic conformal naturality, we obtain the following lemma.

Lemma 4.4. The restriction of the asymptotic barycentric section $\widehat{s}$ to $\widehat{\beta}(A T) \cap$ $\widehat{B}\left(\mathbf{D}^{*}, \Gamma\right)$ gives a real analytic section for $\widehat{\Phi}: \widehat{\operatorname{Bel}}(\mathbf{D}, \Gamma) \rightarrow \widehat{B}\left(\mathbf{D}^{*}, \Gamma\right)$. In particular, $\widehat{\beta}(A T(\Gamma))$ coincides with $\widehat{\beta}(A T) \cap \widehat{B}\left(\mathbf{D}^{*}, \Gamma\right)$, and is the image of $\widehat{\Phi}$.

Proof. Take any $[\varphi]_{B} \in \widehat{\beta}(A T) \cap \widehat{B}\left(\mathbf{D}^{*}, \Gamma\right)$, where $\varphi \in \beta(T)$ satisfies $\gamma^{*} \varphi-\varphi \in$ $B_{0}\left(\mathbf{D}^{*}\right)$ for every $\gamma \in \Gamma$. We consider $\varphi+\psi\left(\psi \in B_{0}\left(\mathbf{D}^{*}\right)\right)$ as an element in the equivalence class $[\varphi]_{B}$. Then, $s(\varphi+\psi)-s(\varphi) \in L_{0}^{\infty}(\mathbf{D})$ by Lemma 4.2. Here, we have that

$$
\gamma^{*}(s(\varphi))-s(\varphi)=s\left(\gamma^{*} \varphi\right)-s(\varphi) \in L_{0}^{\infty}(\mathbf{D}),
$$

where the equality follows from conformal naturality (2) of the barycentric extension e. Hence,

$$
\gamma^{*}(s(\varphi+\psi)) \equiv \gamma^{*}(s(\varphi)) \equiv s(\varphi) \equiv s(\varphi+\psi) \quad\left(\bmod L_{0}^{\infty}(\mathbf{D})\right)
$$

for every $\gamma \in \Gamma$. This implies that $\widehat{s}\left([\varphi]_{B}\right) \in \widehat{\operatorname{Bel}}(\mathbf{D}, \Gamma)$.

Because $\widehat{s}$ is the restriction of the real analytic map to the subspace, it is also real analytic. The restriction of the identity $\widehat{\Phi} \circ \widehat{s}=$ id to $\widehat{\beta}(A T) \cap \widehat{B}\left(\mathbf{D}^{*}, \Gamma\right)$ demonstrates that $\widehat{s}$ is the section for $\widehat{\Phi}: \widehat{\operatorname{Bel}}(\mathbf{D}, \Gamma) \rightarrow \widehat{\Phi}(\widehat{\operatorname{Bel}}(\mathbf{D}, \Gamma))$. In particular, both the inclusions

$$
\widehat{\Phi}(\widehat{\operatorname{Bel}}(\mathbf{D}, \Gamma)) \subset \widehat{\beta}(A T(\Gamma)) \subset \widehat{\beta}(A T) \cap \widehat{B}\left(\mathbf{D}^{*}, \Gamma\right)
$$

are equalities.

From this lemma, we also see that $A T(\Gamma)$ coincides with $\widehat{\pi}(\widehat{\operatorname{Bel}}(\mathbf{D}, \Gamma))$. Now, we are ready to prove the following claim mentioned at the beginning of this section.

Theorem 4.5. The Teichmüller space $A T(\Gamma)$ of $\Gamma$-invariant symmetric structures on $\mathbf{S}$ is contractible.

Proof. The Teichmüller space $A T(\Gamma)$ can be identified with $\widehat{\beta}(A T(\Gamma)) \subset \widehat{B}\left(\mathbf{D}^{*}, \Gamma\right)$, under the asymptotic Bers embedding $\widehat{\beta}$. Because there exists a continuous section $\widehat{s}: \widehat{\beta}(A T(\Gamma)) \rightarrow \widehat{\operatorname{Bel}}(\mathbf{D}, \Gamma)$ by Lemma 4.4 , and because the open unit ball $\widehat{\operatorname{Bel}}(\mathbf{D}, \Gamma)$ of $\widehat{L}^{\infty}(\mathbf{D}, \Gamma)$ is contractible, it follows that $\widehat{\beta}(A T(\Gamma))$ is too. Hence, $A T(\Gamma)$ is contractible. 
The Bers projection $\Phi: \operatorname{Bel}(\mathbf{D}, \Gamma) \rightarrow \beta(T(\Gamma)) \subset B\left(\mathbf{D}^{*}, \Gamma\right)$ is a holomorphic split submersion (see [16, Section 3.4]). As we have mentioned, the asymptotic Bers projection $\widehat{\Phi}: \widehat{\operatorname{Bel}}(\mathbf{D}) \rightarrow \widehat{\beta}(A T) \subset \widehat{B}\left(\mathbf{D}^{*}\right)$ is also a holomorphic split submersion. On the other hand, we do not know whether the restriction of the asymptotic Bers projection $\widehat{\Phi}$ to $\widehat{\operatorname{Bel}}(\mathbf{D}, \Gamma)$ defines a holomorphic submersion $\widehat{\Phi}: \widehat{\operatorname{Bel}}(\mathbf{D}, \Gamma) \rightarrow \widehat{\beta}(A T(\Gamma)) \subset$ $\widehat{B}\left(\mathbf{D}^{*}, \Gamma\right)$ or not. We can only make the following observation.

(1) Because $\widehat{\Phi}: \widehat{\operatorname{Bel}}(\mathbf{D}) \rightarrow \widehat{\beta}(A T)$ is a holomorphic split submersion, we obtain a decomposition of the tangent space

$$
\widehat{L}^{\infty}(\mathbf{D})=\operatorname{Ker}\left(d_{[\mu]_{L}} \widehat{\Phi}\right) \oplus W_{[\mu]_{L}}
$$

at every point $[\mu]_{L} \in \widehat{\operatorname{Bel}}(\mathbf{D}, \Gamma)$, where $W_{[\mu]_{L}}$ is some closed subspace of $\widehat{L}^{\infty}(\mathbf{D})$. Then, the tangent space of $\widehat{\operatorname{Bel}}(\mathbf{D}, \Gamma)$ can be represented by

$$
\widehat{L}^{\infty}(\mathbf{D}, \Gamma)=\operatorname{Ker}\left(\left.d_{[\mu]_{L}} \widehat{\Phi}\right|_{\widehat{L}^{\infty}(\mathbf{D}, \Gamma)}\right) \oplus\left(W_{[\mu]_{L}} \cap \widehat{L}^{\infty}(\mathbf{D}, \Gamma)\right)
$$

Note that $W_{[\mu]_{L}} \cap \widehat{L}^{\infty}(\mathbf{D}, \Gamma)$ is a closed subspace. Hence, if we know that the derivative $\left.d_{[\mu]_{L}} \widehat{\Phi}\right|_{\widehat{L}^{\infty}(\mathbf{D}, \Gamma)}$ at any point is surjective, then we can conclude that the restriction of $\widehat{\Phi}$ to $\widehat{\operatorname{Bel}}(\mathbf{D}, \Gamma)$ is a holomorphic split submersion, by applying the implicit function theorem $([16$, p.89]).

(2) We consider the derivative of $\widehat{\Phi}$ at any point $\widehat{s}\left([\varphi]_{B}\right)$ in the image of the real-analytic section $\widehat{s}: \widehat{\beta}(A T(\Gamma)) \rightarrow \widehat{\operatorname{Bel}}(\mathbf{D}, \Gamma)$. As in the proof of Earle, Markovic, and Saric [5, Proposition 7], a complex linear map on $\widehat{B}\left(\mathbf{D}^{*}, \Gamma\right)$ defined by

$$
[\psi]_{B} \mapsto \frac{1}{2}\left\{d_{[\varphi]_{B}} \widehat{s}\left([\psi]_{B}\right)-i \cdot d_{[\varphi]_{B}} \widehat{s}\left(i[\psi]_{B}\right)\right\}
$$

is a right inverse of $d_{\widehat{s}\left([\varphi]_{B}\right)} \widehat{\Phi}$.

\section{Infinite dimensionality (non-separable)}

In this section, we show that the Teichmüller space $A T(\Gamma)$ of $\Gamma$-invariant symmetric structures on $\mathbf{S}$ for a Fuchsian group $\Gamma$ is of infinite dimension (in fact, it is not separable) unless $T(\Gamma)$ is trivial. This is true even if $T(\Gamma)$ is of finite dimension, and moreover the complement of $\alpha T(\Gamma):=\alpha(T(\Gamma))$ in $A T(\Gamma)$ is not separable if $\Gamma$ is infinite in addition.

To compare $\alpha T(\Gamma)$ with $A T(\Gamma)$, we first verify that $\alpha T(\Gamma)$ is a complex submanifold of $A T(\Gamma)$, and can be identified with $T(\Gamma)$.

Proposition 5.1. Let $\Gamma$ be an infinite Fuchsian group. Then, the projection $\alpha: T \rightarrow A T$ restricted to $T(\Gamma)$ is a biholomorphic embedding, and the image $\alpha T(\Gamma)$ is a complex submanifold of $A T(\Gamma)$.

Proof. Because $P_{B} \circ \beta=\widehat{\beta} \circ \alpha$ on $T$, we can consider these problems in the Bers embeddings. The injection of $\alpha$ on $T(\Gamma)$ follows from the injection of $P_{B}: B\left(\mathbf{D}^{*}\right) \rightarrow$ $\widehat{B}\left(\mathbf{D}^{*}\right)$ on $B\left(\mathbf{D}^{*}, \Gamma\right)$. The latter fact was essentially proved in [13, Theorem 4.2]. A similar statement is also shown in [14, Theorem 2.1]. For the convenience of the reader, we present the proof here.

We have only to show that $B\left(\mathbf{D}^{*}, \Gamma\right) \cap B_{0}\left(\mathbf{D}^{*}\right)=\{0\}$, because of the linearity of $P_{B}$. Take any $\varphi$ in this intersection that satisfies $\gamma^{*} \varphi=\varphi$ for every $\gamma \in \Gamma$, where $\left(\gamma^{*} \varphi\right)(z)=\varphi(\gamma(z)) \gamma^{\prime}(z)^{2}$. Then,

$$
\rho_{\mathbf{D}^{*}}^{-2}(z)|\varphi(z)|=\rho_{\mathbf{D}^{*}}^{-2}(z)\left|\left(\gamma^{*} \varphi\right)(z)\right|=\rho_{\mathbf{D}^{*}}^{-2}(\gamma(z))|\varphi(\gamma(z))| .
$$


Because $\varphi \in B_{0}\left(\mathbf{D}^{*}\right)$, and the infinite Fuchsian group $\Gamma$ contains a sequence $\left\{\gamma_{n}\right\}_{n \in \mathbf{N}}$ such that $\left|\gamma_{n}(z)\right| \rightarrow 1(n \rightarrow \infty)$ for all $z \in \mathbf{D}^{*}$, we have that $\varphi(z) \equiv 0$.

We consider the projection $P_{B}$ of $B\left(\mathbf{D}^{*}, \Gamma\right)$ onto the closed subspace $P_{B}\left(B\left(\mathbf{D}^{*}, \Gamma\right)\right)$ of $\widehat{B}\left(\mathbf{D}^{*}\right)$. Since this is injective as we have seen above, $P_{B}: B\left(\mathbf{D}^{*}, \Gamma\right) \rightarrow P_{B}\left(B\left(\mathbf{D}^{*}, \Gamma\right)\right)$ is an isomorphism between the Banach spaces.

The image of $\alpha T(\Gamma) \subset A T$ under the asymptotic Bers embedding $\widehat{\beta}$ is given by

$$
\widehat{\beta}(\alpha T(\Gamma))=P_{B} \circ \beta(T(\Gamma)) .
$$

Here, $\beta(T(\Gamma))=\beta(T) \cap B\left(\mathbf{D}^{*}, \Gamma\right)$, and $P_{B}$ is an isomorphism of $B\left(\mathbf{D}^{*}, \Gamma\right)$ onto the closed subspace $P_{B}\left(B\left(\mathbf{D}^{*}, \Gamma\right)\right)$ of $\widehat{B}\left(\mathbf{D}^{*}, \Gamma\right)$. Hence, $\widehat{\beta}(\alpha T(\Gamma))$ is a domain in the closed complex submanifold $\widehat{\beta}(A T) \cap P_{B}\left(B\left(\mathbf{D}^{*}, \Gamma\right)\right)$ of $\widehat{\beta}(A T(\Gamma))$. Thus, we see that $\alpha T(\Gamma)$ is a complex submanifold of $A T(\Gamma)$ and $\alpha: T(\Gamma) \rightarrow A T(\Gamma)$ is a biholomorphic embedding.

Remark. In the proof above, it remains a problem whether or not $\widehat{\beta}(\alpha T(\Gamma))$ coincides with $\widehat{\beta}(A T) \cap P_{B}\left(B\left(\mathbf{D}^{*}, \Gamma\right)\right)$.

We expect that the injectivity of $\alpha$ on $T(\Gamma)$, shown in Proposition 5.1, is also valid for a finite Fuchsian group $\Gamma$. However, we do not pursue this question here. Instead, we prove the following claim regarding a finite Fuchsian group, which stands in contrast to the infinite Fuchsian case.

Proposition 5.2. If $\Gamma$ is a finite Fuchsian group, then $A T(\Gamma)=\alpha T(\Gamma)$.

Proof. Denote $\Gamma$ by $\left\{\gamma_{1}, \gamma_{2}, \ldots, \gamma_{m}\right\}$, where $m$ is the order of $\Gamma$. Take an arbitrary point $\alpha \circ \pi(\mu)$ of $A T(\Gamma)$ for $\mu \in \operatorname{Bel}(\mathbf{D})$. The condition that $\alpha \circ \pi(\mu) \in A T(\Gamma)$ is equivalent to $\alpha \circ \pi\left(\gamma_{i}^{*} \mu\right)=\alpha \circ \pi(\mu)$ for every $i=1, \ldots, m$, because $\gamma_{i}^{*} \circ \alpha \circ \pi(\mu)=$ $\alpha \circ \pi\left(\gamma_{i}^{*} \mu\right)$. Then, this is further equivalent to the condition that $P_{L} \circ s \circ \Phi\left(\gamma_{i}^{*} \mu\right)=$ $P_{L} \circ s \circ \Phi(\mu)$, by Lemma 4.2. The conformal naturality (2) of the barycentric extension $e$ implies that

$$
s \circ \Phi\left(\gamma_{i}^{*} \mu\right)=s\left(\gamma_{i}^{*} \Phi(\mu)\right)=\gamma_{i}^{*} \circ s \circ \Phi(\mu) .
$$

Then, we define a Beltrami coefficient

$$
\nu:=\frac{1}{m} \sum_{i=1}^{m} s \circ \Phi\left(\gamma_{i}^{*} \mu\right)=\frac{1}{m} \sum_{i=1}^{m} \gamma_{i}^{*} \circ s \circ \Phi(\mu) .
$$

Moreover, $\nu$ is $\Gamma$-invariant by construction. That is, $\nu \in \operatorname{Bel}(\mathbf{D}, \Gamma)$.

For the proof, we will show that $\alpha \circ \pi(\nu)=\alpha \circ \pi(s \circ \Phi(\mu))$. Then, by the facts that $\alpha \circ \pi(\nu) \in \alpha T(\Gamma)$ and $\pi(s \circ \Phi(\mu))=\pi(\mu)$, we can conclude that $A T(\Gamma)=\alpha T(\Gamma)$. By Proposition 4.1, we only have to show that $P_{L}(\nu)=P_{L}(s \circ \Phi(\mu))$. In other words, $\nu-s \circ \Phi(\mu) \in L_{0}^{\infty}(\mathbf{D})$. Because $s \circ \Phi\left(\gamma_{i}^{*} \mu\right)-s \circ \Phi(\mu) \in L_{0}^{\infty}(\mathbf{D})$ for every $i=1, \ldots, m$, which follows from the condition obtained in the first paragraph, the equality

$$
\nu-s \circ \Phi(\mu)=\frac{1}{m} \sum_{i=1}^{m}\left(s \circ \Phi\left(\gamma_{i}^{*} \mu\right)-s \circ \Phi(\mu)\right)
$$

yields the desired conclusion.

Next, we state the main theorem of this section. The remainder of this section is dedicated to the proof of this theorem.

Theorem 5.3. Assume that an infinite Fuchsian group $\Gamma$ is non-rigid. That is, $T(\Gamma) \neq\{[\mathrm{id}]\}$. Then, $A T(\Gamma)$ contains the submanifold $\alpha T(\Gamma)$ as a proper subset, and is of infinite dimension. More precisely, $A T(\Gamma)-\alpha T(\Gamma)$ is not separable. 
Remark. It is easier to see that $A T(\Gamma)$ itself is not separable when $\operatorname{dim} T(\Gamma)=$ $\infty$, including the case that $\Gamma$ is finite. This is because $T(\Gamma)$ is not separable in this case, but the fibers of the projection $\alpha: T \rightarrow A T$ are always separable.

Proof of Theorem 5.3. Consider the tangent space of $A T(\Gamma)$ at the base point [[id]]. We will choose uncountably many tangent vectors on this tangent space, outside of the tangent subspace of $\alpha T(\Gamma)$. The tangent vectors of $A T$ are represented by the images of the derivative of the projection $\alpha \circ \pi: \operatorname{Bel}(\mathbf{D}) \rightarrow A T$ at 0 , defined on $L^{\infty}(\mathbf{D})$. Moreover, we denote the space of infinitesimally asymptotically trivial Beltrami differentials by

$$
\widehat{N}:=\operatorname{Ker} d_{0}(\alpha \circ \pi) \subset L^{\infty}(\mathbf{D}) .
$$

Correspondingly, the tangent vectors of $T$ are represented by the images of the derivative of the Teichmüller projection $\pi: \operatorname{Bel}(\mathbf{D}) \rightarrow T$ at 0 , defined on $L^{\infty}(\mathbf{D})$, and the space of infinitesimally trivial Beltrami differentials is denoted by $N:=$ Ker $d_{0} \pi \subset L^{\infty}(\mathbf{D})$. Note that by using the global coordinates $\beta: T \rightarrow B\left(\mathbf{D}^{*}\right)$ and $\widehat{\beta}: A T \rightarrow \widehat{B}\left(\mathbf{D}^{*}\right)$, we actually assume that the derivatives $d_{0} \pi$ and $d_{0}(\alpha \circ \pi)$ are identified with the derivatives $d_{0} \Phi$ and $d_{0}\left(P_{B} \circ \Phi\right)$ of the Bers projections, respectively.

The following characterization of $\widehat{N}$ and related facts can be found in the arguments of Earle, Gardiner, and Lakic [3].

Proposition 5.4. (1) A Beltrami differential $\mu \in L^{\infty}(\mathbf{D})$ belongs to $N$ if and only if

$$
\int_{\mathbf{D}} \mu(z) \phi(z) d x d y=0
$$

for every integrable holomorphic function $\phi$ in

$$
A^{1}(\mathbf{D})=\left\{\phi \in \operatorname{Hol}(\mathbf{D})\left|\|\phi\|_{1}=\int_{\mathbf{D}}\right| \phi(z) \mid d x d y<\infty\right\}
$$

(2) $\mu \in L^{\infty}(\mathbf{D})$ belongs to $\widehat{N}$ if and only if

$$
\lim _{n \rightarrow \infty} \int_{\mathbf{D}} \mu(z) \phi_{n}(z) d x d y=0
$$

for every degenerating sequence $\left\{\phi_{n}\right\}_{n \in \mathbf{N}} \subset A^{1}(\mathbf{D})$, which means that $\left\{\phi_{n}\right\}$ converges to 0 locally uniformly on $\mathbf{D}$, and $\left\|\phi_{n}\right\|_{1} \leq 1$ for all $n$;

(3) $\widehat{N}=N+L_{0}^{\infty}(\mathbf{D})$.

Proof. (1) This result is well-known as Teichmüller's lemma. See [1, Section VI.D], [6, Section 6.5], [8, Section 5.7.4], and [16, Section 3.7.1], among other textbooks on Teichmüller spaces. (2) The tangent space $Z=N \backslash L^{\infty}(\mathbf{D})$ of $T$ is isomorphic to the space $H$ of harmonic Beltrami differentials:

$$
H=\left\{\rho_{\mathbf{D}}^{-2}(z) \overline{\phi(z)} \in L^{\infty}(\mathbf{D}) \mid \phi \in B(\mathbf{D})\right\},
$$

where $B(\mathbf{D})$ is the space of hyperbolically bounded holomorphic functions on $\mathbf{D}$. It was proved in [3, Theorem 1.3] that a harmonic Beltrami differential $\mu \in H$ belongs to $L_{0}^{\infty}(\mathbf{D})$ if and only if $\int_{\mathbf{D}} \mu(z) \phi_{n}(z) d x d y \rightarrow 0(n \rightarrow \infty)$ for every degenerating sequence $\left\{\phi_{n}\right\}_{n \in \mathbf{N}} \subset A^{1}(\mathbf{D})$. Because the tangent subspace $Z_{0}=N \backslash L_{0}^{\infty}(\mathbf{D})$ of $Z$ corresponds to the trivial tangent vector of $A T$ via $d_{0} \alpha$, we obtain statement (2). (3) As we have seen in the proof of (2), for $\mu \in H$ the conditions $\mu \in \widehat{N}$ and $\mu \in L_{0}^{\infty}$ (D) are equivalent. Because $L^{\infty}(\mathbf{D})=N \oplus H$, we obtain statement (3). 
Take a small disk $W \subset \mathbf{D}$ centered at 0 such that $\gamma(W) \cap W=\emptyset$ for all $\gamma \in$ $\Gamma-\{$ id $\}$, and set $W_{\gamma}=\gamma(W)$. Because $T(\Gamma) \neq\{[\mathrm{id}]\}$, we can find $\mu_{1} \in L^{\infty}(\mathbf{D}, \Gamma)$ supported on $\bigsqcup_{\gamma \in \Gamma} W_{\gamma}$ such that $\mu_{1} \notin N$. Indeed, the non-rigidity of $T(\Gamma) \neq\{[\mathrm{id}]\}$ is equivalent to a property that there exists a non-trivial holomorphic quadratic differential $\psi$ on $\mathbf{D} / \Gamma$ such that

$$
\int_{\mathbf{D} / \Gamma}|\psi(z)| d x d y<\infty ; \quad \sup _{z \in \mathbf{D} / \Gamma} \rho_{\mathbf{D}}^{-2}(z)|\psi(z)|<\infty .
$$

We restrict the harmonic Beltrami differential $\rho_{\mathbf{D}}^{-2}(z) \overline{\psi(z)} \in L^{\infty}(\mathbf{D}, \Gamma)$ to $\bigsqcup_{\gamma \in \Gamma} W_{\gamma}$, to obtain $\mu_{1} \in L^{\infty}(\mathbf{D}, \Gamma)$. By the surjectivity of the Poincaré series operator (see $[6$, Section 3.2]), there exists an integrable holomorphic function $\widetilde{\psi} \in A^{1}(\mathbf{D})$ such that $\sum_{\gamma \in \Gamma}\left(\gamma^{*} \widetilde{\psi}\right)(z)=\psi(z)$. Hence,

$$
\int_{\mathbf{D}} \mu_{1}(z) \widetilde{\psi}(z) d x d y=\int_{\mathbf{D} / \Gamma} \mu_{1}(z) \psi(z) d x d y=\int_{W} \rho_{\mathbf{D}}^{-2}(z)|\psi(z)|^{2} d x d y>0,
$$

which implies that $\mu_{1} \notin N$, by Proposition 5.4 (1).

Next, we will break the $\Gamma$-invariance of $\mu_{1}$ by introducing weights for $\mu_{1}$ on $\bigsqcup_{\gamma \in \Gamma} W_{\gamma}$. For a function $\xi: \Gamma \rightarrow[0,1]$ on $\Gamma$, we define a Beltrami differential in $L^{\infty}(\mathbf{D})$ by

$$
\mu_{\xi}(z)=\sum_{\gamma \in \Gamma} 1_{W_{\gamma}}(z) \xi(\gamma) \mu_{1}(z)
$$

For any infinite subset $A \subset \mathbf{N}$, the weight function $\xi=\xi_{A}$ is introduced by considering a function $\theta_{A}:[0,+\infty) \rightarrow[0,1]$ that is defined as follows.

For each $n \in \mathbf{N}$, set the interval $[n(n-1), n(n+1))$ by $I_{n}$. If $n \in A$, then $\theta_{A}$ is a tent map on $I_{n}$, which takes a value of 0 at the end points $n(n-1)$ and $n(n+1)$ and of 1 at the mid point $n^{2}$. If $n \notin A$, then $\theta_{A}$ is identically equal to zero on $I_{n}$. This function $\theta_{A}$ is differentiable, except possibly at the points in $\left\{n(n-1), n^{2}\right\}_{n \in \mathbf{N}}$, and satisfies $\theta_{A}^{\prime}(x) \rightarrow 0$ as $x \rightarrow \infty$. The weight function $\xi_{A}$ on $\Gamma$ for $A \subset \mathbf{N}$ is given by

$$
\xi_{A}(\gamma)=\theta_{A}\left(\ell_{\gamma}\right), \quad \ell_{\gamma}=d(\gamma(0), 0)
$$

for $\gamma \in \Gamma$, where $d(\gamma(0), 0)$ is the hyperbolic distance between $\gamma(0)$ and 0 in $\mathbf{D}$.

Note that the set $\left\{\ell_{\gamma}\right\}_{\gamma \in \Gamma}$ is distributed in $[0, \infty)$ without unbounded gaps, in the sense that there exists some constant $L>0$ depending on $\Gamma$ such that every interval of length $L$ in $[0, \infty)$ contains $\ell_{\gamma}$ for some $\gamma \in \Gamma$. To see this, it is sufficient to consider $\left\{\ell_{g^{n}}\right\}_{n \in \mathbf{Z}}$ for any element $g \in \Gamma$ of infinite order. If $g$ is hyperbolic with translation length $t>0$, and the distance to its axis from 0 is $\delta \geq 0$, then $|n| t \leq \ell_{g^{n}} \leq|n| t+2 \delta$. Hence, we can choose $L=t+2 \delta$. If $g$ is parabolic, then $-a+2 \log |n| \leq \ell_{g^{n}} \leq 2 \log |n|+a(n \neq 0)$ for some constant $a>0$ that depends on $d(g(0), 0)$. In this case, we can choose $L=2 a+2$.

Lemma 5.5. The Beltrami differential $\mu_{\xi} \in L^{\infty}(\mathbf{D})$ with the weight function $\xi=\xi_{A}$ for any infinite subset $A \subset \mathbf{N}$ satisfies the following properties:

(1) $\left(\gamma^{*} \mu_{\xi}\right)(z)-\mu_{\xi}(z) \rightarrow 0$ pointwise as $|z| \rightarrow 1$, for every $\gamma \in \Gamma$;

(2) There exist subsequences $\left\{g_{k}\right\}_{k \in \mathbf{N}}$ and $\left\{g_{k}^{\prime}\right\}_{k \in \mathbf{N}}$ in $\Gamma$ such that $\left(g_{k}^{*} \mu_{\xi}\right)(z) \rightarrow$ $\mu_{1}(z)$ and $\left(g^{\prime *} \mu_{\xi}\right)(z) \rightarrow 0$ pointwise as $k \rightarrow \infty$.

Proof. (1) Consider the action of $\Gamma$ on the functions $\xi$ on $\Gamma$, defined by

$$
\left(\gamma^{*} \xi\right)(g)=\xi(\gamma g) \quad(\forall \gamma, g \in \Gamma)
$$


Then, it is easy to see that $\left(\gamma^{*} \mu_{\xi}\right)-\mu_{\xi}=\mu_{\gamma^{*} \xi-\xi}$. Here, for each fixed $\gamma \in \Gamma$, $\left(\gamma^{*} \xi-\xi\right)(g) \rightarrow 0$ as $\ell_{g} \rightarrow \infty$. Indeed, because $\ell$ satisfies $\left|\ell_{\gamma g}-\ell_{g}\right| \leq \ell_{\gamma}$, the condition that $\lim _{x \rightarrow \infty} \theta^{\prime}(x)=0$ implies this property. Note that $\ell_{g} \rightarrow \infty$ as $z \in W_{g}$ approaches S. Hence, we see that $\left(\gamma^{*} \mu_{\xi}\right)(z)-\mu_{\xi}(z)$ tends to zero as $|z| \rightarrow 1$.

(2) We choose a subsequence $\left\{g_{k}\right\} \subset \Gamma$ such that $\theta\left(\ell_{g_{k}}\right) \rightarrow 1$ as $k \rightarrow \infty$. This is possible because every interval of a certain length $L>0$ in $[0, \infty)$ contains $\ell_{g}$ for some $g \in \Gamma$, as we have seen above. Because $\left|\ell_{g_{k} \gamma}-\ell_{g_{k}}\right| \leq \ell_{\gamma}$, we also have that $\theta\left(g_{k} \gamma\right) \rightarrow 1$ as $k \rightarrow \infty$ for each fixed $\gamma \in \Gamma$. This implies that $g_{k}^{*} \xi(\gamma) \rightarrow 1$, and hence $\left(g_{k}^{*} \mu_{\xi}\right)(z) \rightarrow \mu_{1}(z)$ pointwise as $k \rightarrow \infty$. Note that $g_{k}^{*} \mu_{\xi}=\mu_{g_{k}^{*} \xi}$. Similarly, if we choose a subsequence $\left\{g_{k}^{\prime}\right\} \subset \Gamma$ such that $\theta\left(\ell_{g_{k}^{\prime}}\right) \rightarrow 0$ as $k \rightarrow \infty$, then we obtain the second assertion.

Property (1) of the above lemma implies that $\gamma^{*} \mu_{\xi}-\mu_{\xi} \in \widehat{N}$ for every $\gamma \in \Gamma$. This is equivalent to saying that $d_{0}(\alpha \circ \pi)\left(\mu_{\xi}\right)$ lies on the tangent space of $A T(\Gamma)$. On the other hand, property (2) implies that $\mu_{\xi}$ is not equivalent to any $\Gamma$-invariant vector $\nu \in L^{\infty}(\mathbf{D}, \Gamma)$ modulo $\widehat{N}$. Equivalently, $d_{0}(\alpha \circ \pi)\left(\mu_{\xi}\right)$ does not lie on the tangent space of $\alpha T(\Gamma)$. In the next proposition, we will verify these consequences from Lemma 5.5. This constitutes a refinement of our previous arguments in [10, 11].

Proposition 5.6. The Beltrami differential $\mu_{\xi}$ satisfies the following properties: (1) $d_{0}(\alpha \circ \pi)\left(\mu_{\xi}\right)$ is $\Gamma$-invariant as a tangent vector of $A T ;(2) d_{0}(\alpha \circ \pi)\left(\mu_{\xi}\right)$ does not lie on the tangent space of $\alpha T(\Gamma)$. Hence, $d_{0}(\alpha \circ \pi)\left(\mu_{\xi}\right)$ is a tangent vector of $A T(\Gamma)$, but not of $\alpha T(\Gamma)$.

Proof. (1) Lemma 5.5 (1) implies that $\gamma^{*} \mu_{\xi}-\mu_{\xi} \in L_{0}^{\infty}(\mathbf{D})$ for every $\gamma \in \Gamma$. Hence,

$$
\gamma^{*}\left(d_{0}(\alpha \circ \pi)\left(\mu_{\xi}\right)\right)=d_{0}(\alpha \circ \pi)\left(\gamma^{*} \mu_{\xi}\right)=d_{0}(\alpha \circ \pi)\left(\mu_{\xi}\right)
$$

by Proposition 5.4 (3), which implies $\Gamma$-invariance.

(2) Suppose on the contrary that we can write $\mu_{\xi}=\nu+\lambda$, where $\nu \in L^{\infty}(\mathbf{D}, \Gamma)$ and $\lambda \in \widehat{N}$. Let $\left\{g_{k}\right\}_{k \in \mathbf{N}} \subset \Gamma$ be the sequence chosen in Lemma 5.5 (2). Take any $\phi \in A^{1}(\mathbf{D})$ with $\|\phi\|_{1}=1$, and set $\phi_{k}=\left(g_{k}\right)_{*} \phi:=\left(g_{k}^{-1}\right)^{*} \phi$ for every $k \in \mathbf{N}$. Then, $\left\{\phi_{k}\right\}_{k \in \mathbf{N}}$ is a degenerating sequence, which can be seen from the fact that $\Gamma$ is discrete and considering the inclusion $A^{1}(\mathbf{D}) \subset B_{0}(\mathbf{D})$, where $B_{0}(\mathbf{D})$ is defined similarly to $B_{0}\left(\mathbf{D}^{*}\right)$. This inclusion is well-known. We refer the reader to [14, Proposition 5.3] for a simpler proof.

Because $\nu$ is $\Gamma$-invariant, we have that

$\int_{\mathbf{D}}\left(g_{k}^{*} \mu_{\xi}\right)(z) \phi(z) d x d y=\int_{\mathbf{D}} \mu_{\xi}(z) \phi_{k}(z) d x d y=\int_{\mathbf{D}} \nu(z) \phi(z) d x d y+\int_{\mathbf{D}} \lambda(z) \phi_{k}(z) d x d y$.

Because $\left(g_{k}^{*} \mu_{\xi}\right)(z) \rightarrow \mu_{1}(z)$ as $k \rightarrow \infty$ by Lemma $5.5(2)$, the left side of the above equality converges to $\int_{\mathbf{D}} \mu_{1}(z) \phi(z) d x d y$, by the dominated convergence theorem. On the other hand, $\int_{\mathbf{D}} \lambda(z) \phi_{k}(z) d x d y$ converges to 0 by Proposition 5.4 (2), because $\lambda \in \widehat{N}$, and $\left\{\phi_{k}\right\}$ is a degenerating sequence. Hence,

$$
\int_{\mathbf{D}} \mu_{1}(z) \phi(z) d x d y=\int_{\mathbf{D}} \nu(z) \phi(z) d x d y
$$

for every $\phi \in A^{1}(\mathbf{D})$ with $\|\phi\|_{1}=1$, which implies that $\mu_{1}-\nu \in N \subset \widehat{N}$. From this and the fact that $\mu_{\xi}=\nu+\lambda$, we have that $\mu_{1}-\mu_{\xi}=\lambda^{\prime}$, for some other $\lambda^{\prime} \in \widehat{N}$.

Next, we set $\phi_{k}^{\prime}=\left(g_{k}^{\prime}\right)_{*} \phi$ for every $k \in \mathbf{N}$, and consider another degenerating sequence $\left\{\phi_{k}^{\prime}\right\}_{k \in \mathbf{N}}$. Similarly to the above paragraph, and by the fact that $\mu_{1}$ is 
$\Gamma$-invariant, we have that

$\int_{\mathbf{D}}\left(\mu_{1}(z)-\left(g_{k}^{\prime *} \mu_{\xi}\right)(z)\right) \phi(z) d x d y=\int_{\mathbf{D}}\left(\mu_{1}(z)-\mu_{\xi}(z)\right) \phi_{k}^{\prime}(z) d x d y=\int_{\mathbf{D}} \lambda^{\prime}(z) \phi_{k}^{\prime}(z) d x d y$.

Because $\left(g_{k}^{\prime *} \mu_{\xi}\right)(z) \rightarrow 0$ as $k \rightarrow \infty$ by Lemma $5.5(2)$, the left side of the above equality converges to $\int_{\mathbf{D}} \mu_{1}(z) \phi(z) d x d y$ and the right side to 0 as $k \rightarrow \infty$. Hence, $\int_{\mathbf{D}} \mu_{1}(z) \phi(z) d x d y=0$ for every such $\phi \in A^{1}(\mathbf{D})$, which implies that $\mu_{1} \in N$ by Proposition 5.4 (1). However, this contradicts the manner of choosing $\mu_{1}$.

Next, we construct uncountably many such Beltrami differentials $\mu_{\xi} \in L^{\infty}(\mathbf{D})$ for $\xi=\xi_{A}$ independently, by choosing a suitable uncountable family of infinite subsets $A \subset \mathbf{N}$. More precisely, for an uncountable index set $J$, the family $\left\{A_{j}\right\}_{j \in J}$ of infinite subsets $A_{j}$ is required to satisfy the condition that the symmetric difference $A_{j} \ominus A_{j^{\prime}}$ is also infinite for any distinct $j, j^{\prime} \in J$. The existence of such a family is easily seen.

For $A_{j}$ and $A_{j^{\prime}}\left(j \neq j^{\prime}\right)$ as above, set $\xi_{j}=\xi_{A_{j}}$ and $\xi_{j^{\prime}}=\xi_{A_{j^{\prime}}}$, and consider $\mu_{\xi_{j}}$ and $\mu_{\xi_{j^{\prime}}}$. Take some $\phi \in A^{1}(\mathbf{D})$, with $\|\phi\|_{1}=1$, such that $\int_{\mathbf{D}} \mu_{1}(z) \phi(z) d x d y \neq 0$. Because $A_{j} \ominus A_{j^{\prime}}$ is infinite, we may assume that $A_{j}-A_{j^{\prime}}$ is also infinite, and denote the positive integers in this set by $\left\{n_{k}\right\}_{k \in \mathbf{N}}$. For each $k \in \mathbf{N}$, choose $g_{k} \in \Gamma$ such that $\left|\ell_{g_{k}}-n_{k}^{2}\right| \leq L / 2$, where $L>0$ is the constant defined just prior to Lemma 5.5 by the distribution of the orbit $\Gamma(0)$. Then, we set $\phi_{k}=\left(g_{k}\right)_{*} \phi$, and construct a degenerating sequence $\left\{\phi_{k}\right\}_{k \in \mathbf{N}}$.

Proposition 5.7. Under the circumstances given above,

$$
\lim _{k \rightarrow \infty} \int_{\mathbf{D}}\left(\mu_{\xi_{j}}(z)-\mu_{\xi_{j^{\prime}}}(z)\right) \phi_{k}(z) d x d y=\int_{\mathbf{D}} \mu_{1}(z) \phi(z) d x d y \neq 0
$$

for any distinct $j, j^{\prime} \in J$.

Proof. As before, we have that

$\int_{\mathbf{D}}\left(\mu_{\xi_{j}}(z)-\mu_{\xi_{j^{\prime}}}(z)\right) \phi_{k}(z) d x d y=\int_{\mathbf{D}} g_{k}^{*} \mu_{\xi_{j}-\xi_{j^{\prime}}}(z) \phi(z) d x d y=\int_{\mathbf{D}} \mu_{g_{k}^{*}\left(\xi_{j}-\xi_{j^{\prime}}\right.}(z) \phi(z) d x d y$.

Here, $g_{k}^{*}\left(\xi_{j}-\xi_{j^{\prime}}\right)$ converges to the constant function 1 as $k \rightarrow \infty$, and hence $\mu_{g_{k}^{*}\left(\xi_{j}-\xi_{j^{\prime}}\right)}(z)$ converges to $\mu_{1}(z)$ pointwise. Taking the limit using the dominated convergence theorem yields the stated assertion.

As a generalization of Proposition 5.4 (2), Earle, Gardiner, and Lakic [3, Section 3.1] proved that the norm of the tangent vectors on $A T$ can be defined by

$$
\sup _{\left\{\phi_{k}\right\}} \limsup _{k \rightarrow \infty}\left|\int_{\mathbf{D}} \mu(z) \phi_{k}(z) d x d y\right|
$$

where the supremum is taken over all degenerate sequences $\left\{\phi_{k}\right\}_{k \in \mathbf{N}} \subset A^{1}(\mathbf{D})$. Then, Proposition 5.7 verified above shows that the uncountable family of tangent vectors $\left\{d_{0}(\alpha \circ \pi)\left(\mu_{\xi_{j}}\right)\right\}_{j \in J}$ is a discrete set on the tangent space of $A T(\Gamma)$, which is not in the tangent space of $\alpha T(\Gamma)$. This implies the non-separability of $A T(\Gamma)-\alpha T(\Gamma)$. Hence, the proof of Theorem 5.3 is completed.

Remark. When a Fuchsian group $\Gamma$ is rigid, we make no claims regarding $A T(\Gamma)$ in Theorem 5.3. We have also investigated a certain strategy for demonstrating the non-triviality of $A T(\Gamma)$ in this case, in our former work [12]. However, we did not reach the conclusion there. Therefore, it may be meaningful to consider the problem of whether or not $A T(\Gamma)=\{[[\mathrm{id}]]\}$ when $T(\Gamma)=\{[\mathrm{id}]\}$. If $T(\Gamma)=\{[\mathrm{id}]\}$ implies 
$A T(\Gamma)=\{[[\mathrm{id}]]\}$, then this rigidity is not only holds for the deformation of $\Gamma$ in $\operatorname{Möb}(\mathbf{S})$, but also for the deformation in Sym.

\section{Group invariant symmetric structures on a Riemann surface}

In this supplementary section, we denote our Teichmüller space $A T(\Gamma)$ for a Fuchsian group $\Gamma$ by $A T(\mathbf{D}, \Gamma)$. Furthermore, we denote the asymptotic Teichmüller space $A T(R)$ of a hyperbolic Riemann surface $R$ by $A T(R, 1)$. The definition of the latter space can be found in $[3,4,5,6]$. Then, we can unify these two generalizations of $A T=A T(\mathbf{D}, 1)$ by using the notation $A T(R, G)$, where $R$ is a hyperbolic Riemann surface and $G$ is a subgroup of the $\operatorname{group} \operatorname{Aut}(R)$ of conformal automorphisms of $R$. We call $A T(R, G)$ the $G$-invariant asymptotic Teichmüller space of $R$. Note that $A T(R)$ consists of a single point whenever $R$ is cofinite. In this case, $\operatorname{Aut}(R)$ is a finite group and $A T(R, G)$ is trivial.

Theorem 6.1. For a hyperbolic Riemann surface $R$ and an infinite subgroup $G \subset \operatorname{Aut}(R)$, the $G$-invariant asymptotic Teichmüller space $A T(R, G)$ of $R$, endowed with the asymptotic Teichmüller distance, admits a complex non-separable Banach manifold structure if $T(R / G)$ is non-trivial. Moreover, $A T(R, G)$ is contractible.

The proof can be obtained in almost the same manner as in the case of $R=\mathbf{D}$. Note that the results concerning the asymptotic Teichmüller space $A T$ that we used in the previous arguments are also valid for the asymptotic Teichmüller space $A T(R)$ of $R$.

\section{References}

[1] Ahlfors, L.: Lectures on quasiconformal mappings. - Van Nostrand, 1966.

[2] Doundy, A., and C. J. Earle: Conformally natural extension of homeomorphisms of the circle. - Acta Math. 157, 1986, 23-48.

[3] Earle, C. J., F. P. Gardiner, and N. Lakic: Asymptotic Teichmüller space, Part I: The complex structure. - In the tradition of Ahlfors and Bers, Contemporary Math. 256, Amer. Math. Soc., 2000, 17-38.

[4] Earle, C. J., F. P. Gardiner, and N. Lakic: Asymptotic Teichmüller space, Part II: The metric structure. - In the tradition of Ahlfors and Bers III, Contemporary Math. 355, Amer. Math. Soc., 2004, 187-219.

[5] Earle, C. J., V. Markovic, and D. Saric: Barycentric extension and the Bers embedding for asymptotic Teichmüller space. - In: Complex manifolds and hyperbolic geometry, Contemporary Math. 311, Amer. Math. Soc., 2002, 87-105.

[6] Gardiner, F.P., and N. Lakic: Quasiconformal Teichmüller theory. - Math. Surveys Monogr. 76, Amer. Math. Soc., 2000.

[7] Gardiner, F. P., and D. P. Sullivan: Symmetric structure on a closed curve. - Amer. J. Math. 114, 1992, 683-736.

[8] Leнto, O.: Univalent functions and Teichmüller spaces. - Grad. Texts in Math. 109, Springer, 1986.

[9] Matsuzaki, K.: Inclusion relations between the Bers embeddings of Teichmüller spaces. Israel J. Math. 140, 2004, 113-124.

[10] Matsuzaki, K.: Symmetric groups that are not the symmetric conjugates of Fuchsian groups. - In the tradition of Ahlfors-Bers V, Contemporary Math. 510, Amer. Math. Soc., 2010, 239 247. 
[11] Matsuzaki, K.: The action of elliptic modular transformations on asymptotic Teichmüller spaces. - In: Teichmüller theory and moduli problem, Ramanujan Math. Soc. Lect. Notes Ser. 10, Ramanujan Math. Soc., 2010, 481-488.

[12] MatsuZaki, K.: An averaging operator and non-separability of certain Banach spaces of holomorphic automorphic forms. - In: Infinite dimensional Teichmüller spaces and moduli spaces, RIMS Kokyuroku Bessatsu B17, Res. Inst. Math. Sci., 2010, 65-72.

[13] Matsuzaki, K.: Properties of asymptotically elliptic modular transformations of Teichmüller spaces. - In: Infinite dimensional Teichmüller spaces and moduli spaces, RIMS Kokyuroku Bessatsu B17, Res. Inst. Math. Sci., 2010, 73-84.

[14] Matsuzaki, K.: Rigidity of groups of circle diffeomorphisms and Teichmüller spaces. arXiv:1607.06316.

[15] McMullen, C.: Amenability, Poincaré series and quasiconformal maps. - Invent. Math. 97, 1989, 95-127.

[16] NAG, S.: The complex analytic theory of Teichmüller spaces. - John Wiley \& Sons, 1988.

Received 19 August 2016• Accepted 9 September 2016 